\title{
Electrostrictive deformations in small carbon clusters, hydrocarbon molecules, and carbon nanotubes
}

\author{
I. Cabria, ${ }^{1}$ C. Amovilli, ${ }^{2}$ M. J. López, ${ }^{1}$ N. H. March, ${ }^{3}$ and J. A. Alonso ${ }^{1}$ \\ ${ }^{1}$ Departamento de Física Teórica, Universidad de Valladolid, 47005 Valladolid, Spain \\ ${ }^{2}$ Dipartimento di Chimica e Chimica Industriale, Università di Pisa, Via Risorgimento 35, 56126 Pisa, Italy \\ ${ }^{3}$ Department of Physics, University of Antwerp, B-2020 Antwerp, Belgium and Oxford University, Oxford, United Kingdom
}

(Received 5 September 2006; published 6 December 2006)

\begin{abstract}
The electrostrictive response of small carbon clusters, hydrocarbon molecules, and carbon nanotubes is investigated using the density functional theory. For ringlike carbon clusters, one can get insight on the deformations induced by an electric field from a simple two-dimensional model in which the positive charge of the carbon ions is smeared out in a circular homogeneous line of charge and the electronic density is calculated for a constant applied electric field within a two-dimensional Thomas-Fermi method. According to the Hellmann-Feynman theorem, this model predicts, for fields of about $1 \mathrm{~V} / \AA$, only a small elongation of the ring clusters in the direction of the electric field. Full three-dimensional density functional calculations with an external electric field show similar small deformations in the ring carbon clusters compared to the simple model. The saturated benzene and phenanthrene hydrocarbon molecules do not experience any deformation, even under the action of relatively intense $(1 \mathrm{~V} / \AA)$ electric fields. In contrast, finite carbon nanotubes experience larger elongations $(\sim 2.9 \%)$ induced by relatively weak $(0.1 \mathrm{~V} / \AA)$ applied electric fields. Both C-C bond length elongation and the deformation of the honeycomb structure contribute equally to the nanotube elongation. The effect of the electric field in hydrogen terminated nanotubes is reduced with respect to the nanotubes with dangling bonds in the edges.
\end{abstract}

DOI: 10.1103/PhysRevA.74.063201

PACS number(s): 36.40.Mr, 42.50.Vk, 73.22.-f

\section{INTRODUCTION}

Ferroelectric and electrostrictive materials are able to convert electrical energy into mechanical energy. Carbon nanotubes (CNTs) are highly sensitive to applied electric fields [1-6] and the use of CNTs as electromechanical actuators has been proposed recently [7]. Although the electrostrictive effect is less intense in CNTs than in other electrostrictive materials $[8,9]$, the advantages of CNTs are their nanometer size, their mechanical properties, the absence of fatigue and degradation, and the fact that they can support high voltages without breaking down. On the other hand, since the volumetric and gravimetric work densities of the electrostrictive materials are proportional to the Young's modulus, it has been predicted [10] that CNTs would have work densities of three and six orders of magnitude higher than those of the ferroelectric and polymer electrostrictive materials, due to their high Young's modulus, in the region of TPas.

Exceptionally large electrostrictive deformations have been predicted by Guo and Guo for carbon nanotubes of finite length based on Hartree-Fock and density functional theory (DFT) calculations [10]. The calculated axial deformations are about $12 \%$ in finite $(3,3)$ and $(5,0)$ carbon nanotubes under fields of $0.4 \mathrm{~V} / \AA ̊$. Much smaller elongations (about 2.6\%) are obtained for the corresponding unwrapped finite planar graphene sheets. In a $(5,5)$ CNT capped with a $\mathrm{C}_{30}$ cluster at one end and saturated with hydrogen atoms at the other end the intensity of the electric field has to be increased up to $1 \mathrm{~V} / \AA$ for having an electrostrictive deformation of about $12 \%$, similar to that in open-end nanotubes. These results indicate that a larger electric field is necessary to get similar deformations in nanotubes with no dangling bonds at the ends as compared to open-end nanotubes (with dangling bonds at the tube edge). In all cases, the nanotube elongations are attributed to the change in the C-C bond lengths and the authors claim that there is no deformation of the honeycomb network of the nanotube. Also, charge polarization of the nanotubes has been obtained upon application of an electric field [11].

On the other hand, DFT calculations of field emission (FE) from clean edge and H-terminated graphitic ribbons show very different FE currents [12]. The dangling bond states localized at clean edges are the major contributors to the FE current. The differences, therefore, arise from the disappearance of dangling bond states at the edges of the $\mathrm{H}$-terminated ribbons. Ab initio calculations on the unraveling of double wall zig-zag CNTs predict that the tubes can withstand electric field strengths of up to $2 \mathrm{~V} / \AA$ before they begin to disintegrate from the exposed edge [13]. However, an unstable failure is obtained at fields as low as $0.36 \mathrm{~V} / \AA$ for a finite $(5,0)$ unwrapped planar graphene sheet [10]. Recently, it has been demonstrated that atomic force microscopy in contact mode can be used to determine the electromechanical response of bundles of single wall CNTs [14]. The measured electrostrictive strain (change in the bundle thickness) is about $2.6 \%$ under an applied electric field of $0.016 \mathrm{~V} / \AA$. This electrostrictive thickness deformation of the bundles is about one order of magnitude larger than the calculated [10] axial deformation of individual nanotubes. However, the electrostrictive behavior along the axial direction of the nanotubes was not measured.

Due to the lack of experimental studies on the axial electrostrictive behavior of CNTs and on the role played by the dangling bonds and the hydrogen saturated bonds in the deformation of the tubes, additional theoretical investigations are required to shed light into this problem. In this paper, we 
concentrate on small pure carbon and hydrocarbon systems, including CNTs. The present study has two parts. First we apply a simple two-dimensional Thomas-Fermi method to study ringlike carbon clusters which have been modeled by a uniform ring of positive charge in a two-dimensional electronic cloud. This model has been worked out for zero electric field by two of us [15], and here the model is extended to study the clusters under an applied electric field. In the presence of an external electric field of $1 \mathrm{~V} / \AA$ the circular clusters deform very little, suffering only a small elongation of their shape with the symmetry axis along the direction of the applied electric field. Second, using full three-dimensional density functional theory, we calculate the electrostrictive response of small ring carbon clusters, hydrocarbon molecules, and finite CNTs with and without hydrogen-saturated edges. The effect of the electric field on the hydrocarbon molecules and on the CNTs with hydrogen-saturated edges is smaller than on the corresponding systems without hydrogensaturated edges; it produces moderate deformations in the small carbon clusters and a larger elongation in the open-end (with no saturated edges) finite CNTs.

\section{TWO-DIMENSIONAL THOMAS-FERMI MODEL OF RINGLIKE CARBON CLUSTERS IN AN ELECTRIC FIELD}

There is experimental evidence [16] that small neutral carbon clusters $\mathrm{C}_{4 n+2}(n \geqslant 2)$ produced by the laser ablation technique present monocyclic ring structures. Two of us have recently [15] extended the so-called March model of fullerenes [17-19] to treat carbon clusters with ring structure. The ring carbon clusters are modeled by a circular line of homogeneous positive charge obtained by smearing out the effective positive charge (charge $f$ per ion) of the $N$ carbon ions of the ring cluster. A two-dimensional Thomas-Fermi method is then applied to obtain the self-consistent electronic density and the corresponding self-consistent electrostatic potential $V_{\mathrm{es}}$. Putting the chemical potential $\mu$ equal to zero, and using a.u., the electrostatic potential is given by

$$
\begin{aligned}
V_{\mathrm{es}}(r) & =-\pi \rho(r) \\
& =-\lambda\left[K_{0}(t) \Theta(t-s) I_{0}(s)+I_{0}(t) \Theta(s-t) K_{0}(s)\right],
\end{aligned}
$$

where $K_{0}$ and $I_{0}$ are modified Bessel functions, $\Theta$ is the Heaviside step function, $t=2 R$, and $s=2 r$, where $R$ is the radius of the carbon ring and $r$ is the radial distance from the cluster center. $\lambda=2 N f$ is obtained from the condition of electron density normalization to the value $N f$. In this model the charge $f$ per ion is the number of valence electrons which are assumed to be delocalized on the plane of the ring. It cannot be greater than 4 for carbon atoms. The calculated selfconsistent electrostatic potential, scaled by $\lambda, \quad v(s)$ $=V_{\text {es }}(s) / \lambda$, to eliminate its dependence in $N$ and $f$, is plotted in Fig. 1 for various values of the scaled ring radius $t$.

To investigate, qualitatively, the structural deformations of ring carbon clusters induced by an external electric field we have extended the two-dimensional Thomas-Fermi (TF) model to incorporate an external electric field. The chemical potential of a two-dimensional ring cluster lying in the $y z$

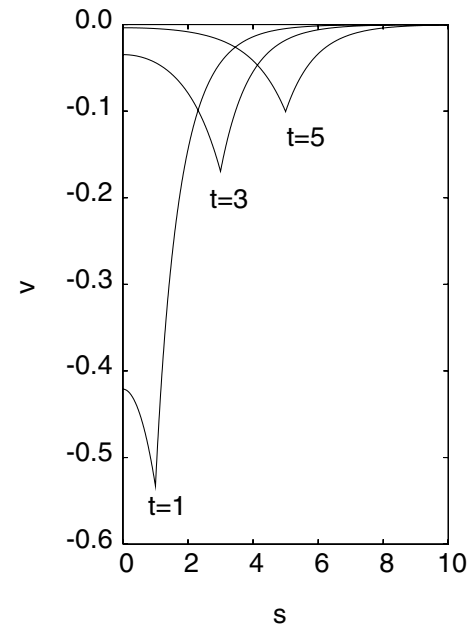

FIG. 1. Scaled self-consistent Thomas-Fermi potential $v(s)$ for a two-dimensional carbon cluster where the positive ionic charge has been smeared out on a circular line of charge, as a function of a properly scaled distance $s$ from the cluster center and for different values of the scaled ring radius $t$. Scaled units are specified in the text.

plane under the action of a constant electric field of strength $\epsilon$ applied in the $z$ direction is given by

$$
\mu=2 c_{k}^{(2)} \rho(y, z)+V_{\mathrm{es}}(y, z)+\epsilon z+\left(k_{y} y^{2}+k_{z} z^{2}\right) / 2,
$$

where $c_{k}^{(2)}=\pi / 2$ is the constant, in a.u., in the kinetic energy functional

$$
T_{s}=c_{k}^{(2)} \int \rho^{2} d y d z .
$$

One should notice that a harmonic term has been added in the equation of the chemical potential, to keep the electron density confined, thereby preventing field ionization. The electronic density $\rho$ is calculated using in Eq. (2) the electrostatic potential of the field-free cluster given by Eq. (1). On the other hand, since the potential $V_{\text {es }}$ used in Eq. (2) is that of the system without electric field, the calculated density is non-self-consistent. In other words, the external electric field has been considered as a perturbation over the electrostatic field of the free cluster.

Some contours of constant areal electron density (number of electrons per unit area) are shown in Fig. 2. The plot corresponds to a ring of $N=6$, radius $=1.23 \AA$ (the equilibrium radius of the $\mathrm{C}_{6}$ cluster in the DFT calculations by Amovilli and March [15]), $f=2.7, k_{y}=k_{z}=0.03$ a.u. (harmonic force constants), and an applied electric field of intensity $\epsilon=0.019$ a.u. $=1 \mathrm{~V} / \AA$ along the $z$ axis. Amovilli and March found in the above-mentioned paper [15] that $f=2.7$ allows the model to reproduce $a b$ initio equilibrium radii once an effective nuclear interaction has been introduced.

The harmonic force constants are free parameters in the present TF model, and hence the choice of $k_{y}$ and $k_{z}$ determines the value of the chemical potential. In the present conditions the chemical potential has a value of -0.017 a.u. The contours plotted represent densities between 0 and 0.1 in a.u. The most external contour is that for $\rho=0$, while the 


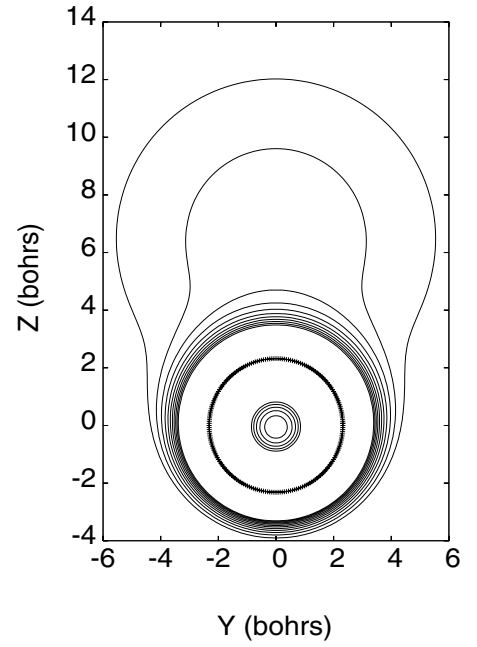

FIG. 2. Contours of constant electron density (in a.u., $e /$ bohr $^{2}$ ) for the two-dimensional Thomas-Fermi model of a $\mathrm{C}_{6}$ ring cluster, in the presence of an electric field of magnitude 0.019 a.u. $=1 \mathrm{~V} / \AA$ along the $z$ axis and harmonic confinement, obtained from Eqs. (1) and (2). From outside to inside, the density of the first ten contours increases from 0 to 0.1 in steps of 0.01 a.u.

density of the other contours increases in steps of 0.01 a.u. The two outermost contours, corresponding to the two lowest density values, have a marked "pearlike" shape, with the density elongated in the direction of the applied electric field. But, as the density increases, the contours become nearly circular quickly, showing only a small percent elongation. Contours of density higher than 0.1 a.u., not plotted in the figure, have a very small distortion. This occurs in particular for the contour $\rho=1.1$ a.u., which lies practically on the ring of the smeared nuclear charge.

The Hellmann-Feynman theorem tells us that, at equilibrium, the atomic nuclei sit at points of zero electric field. We then expect the ring of positive charge to mimic approximately the shape of the high density contours and to suffer a very small deformation. This model, therefore, predicts, for fields of 0.019 a.u. $=1 \mathrm{~V} / \AA$, a very small deformation of the shape of ring clusters along the direction of the applied electric field. It will be shown in the next section that full threedimensional DFT calculations confirm the qualitative predictions of the simple two-dimensional Thomas-Fermi model.

\section{DENSITY FUNCTIONAL CALCULATIONS OF THE EFFECT OF AN APPLIED ELECTRIC FIELD}

The density functional formalism has been used to investigate the electrostrictive response of small carbon clusters, hydrocarbon molecules, and finite CNTs. We have used the DACAPO code [20]. The code utilizes supercells, a basis set of plane waves [21], and ultrasoft pseudopotentials [22]. For the exchange and correlation functional we have used the generalized gradient approximation of Perdew and Wang [23]. The code allows for the application of a constant electric field along a specific direction by introducing an external dipole layer, placed on both sides of the molecule and far from it.

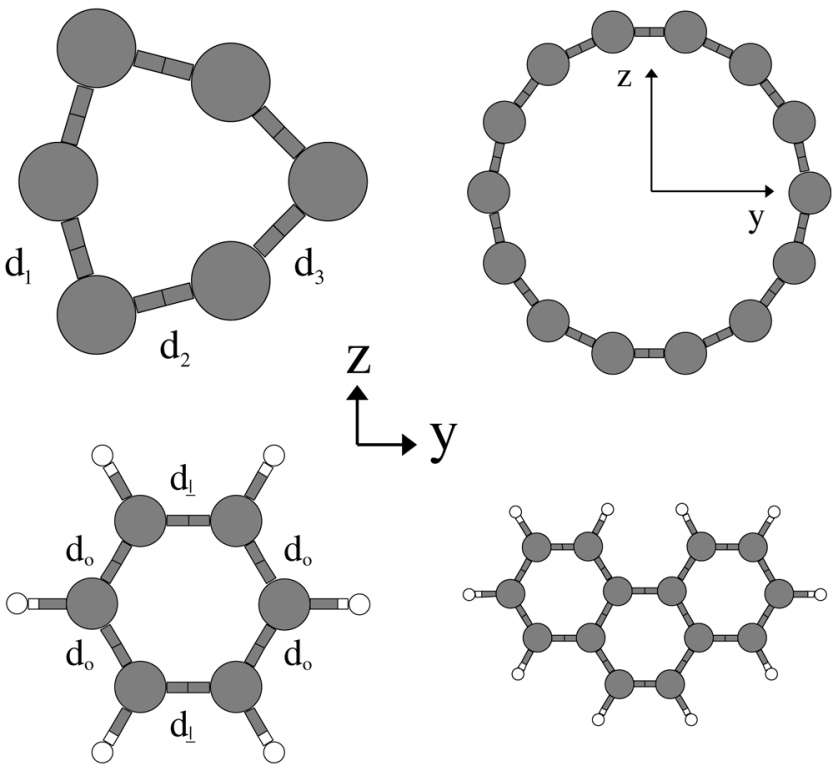

FIG. 3. Optimized geometries of the $\mathrm{C}_{6}$ cluster with $D_{3 h}$ symmetry, $\mathrm{C}_{14}$ cluster ring, and the benzene $\left(\mathrm{C}_{6} \mathrm{H}_{6}\right)$ and phenanthrene $\left(\mathrm{C}_{14} \mathrm{H}_{10}\right)$ molecules.

\section{A. Optimized nuclear geometries without an electric field}

This formalism has been used to optimize the ringlike geometries of the $\mathrm{C}_{6}$ and the $\mathrm{C}_{14}$ pure carbon clusters and of the related benzene $\left(\mathrm{C}_{6} \mathrm{H}_{6}\right)$ and phenanthrene $\left(\mathrm{C}_{14} \mathrm{H}_{10}\right)$ hydrocarbon molecules, in free space and in the presence of an applied electric field of $1 \mathrm{~V} / \AA$. The optimized geometries in the absence of the electric field are plotted in Fig 3. The lowest energy structure of $\mathrm{C}_{6}$ predicted by quantum chemical [24] and DFT [25] calculations is a cyclic ring with $D_{3 h}$ symmetry. The linear chain isomer comes out higher in energy. We have fully optimized the ring structure of $\mathrm{C}_{6}$ and also obtained the $D_{3 h}$ symmetry, with geometrical parameters very close to those reported by Jones [25]. The $\mathrm{C}-\mathrm{C}$ and $\mathrm{C}-\mathrm{H}$ bond distances (1.40 and $1.09 \AA$, respectively) that we obtain for the optimized structure of the benzene molecule perfectly reproduce the experimental values. The calculated minimum energy structure of the $\mathrm{C}_{14}$ cluster is the monocyclic ring with all the $\mathrm{C}-\mathrm{C}$ bond lengths equal, in agreement with previous DFT studies [25,26] and mass spectroscopy experiments [16]. The structure of the carbon network of the phenanthrene molecule (that is, without the $\mathrm{H}$ atoms) has been proposed $[25,26]$ as a high energy isomer of $\mathrm{C}_{14}$, but this is not a stable geometry and it relaxes toward the ring structure. Similarly, the distorted ring with the shape of an ellipse relaxes toward the circular ring.

\section{B. Electrostrictive response of $\mathrm{C}$ clusters and small polyacenes}

The electrostrictive deformations of the structures of the $\mathrm{C}_{6}$ and $\mathrm{C}_{14}$ clusters and of the benzene and phenanthrene molecules upon application of an intense electric field of $1 \mathrm{~V} / \AA$ are given in Table I. Relative changes in the overall length of the molecules are given in two orthogonal directions in the molecular plane ( $y$ and $z$ directions), one of which is, in fact, the direction of the applied field. The first 
TABLE I. Deformations induced by an applied electric field on $\mathrm{C}_{6}$ and $\mathrm{C}_{14}$, on the carbon network of benzene and phenanthrene, on a clean finite $(4,4)$ carbon nanotube with 48 atoms and on the same nanotube with edges saturated by hydrogen atoms. The field has strengths of $0.1 \mathrm{~V} / \AA$ for the CNT and $1 \mathrm{~V} / \AA$ for the other clusters and molecules. Geometries and field directions are shown in Figs. 3 and 4 . $\mathcal{E}(a)$ represents the relative change (in \%) in quantity $a . Y$ and $Z$ are the lengths of the molecules in the $y$ and $z$ directions, respectively. $d_{1}, d_{2}$ and $d_{3}$ are bond lengths of $\mathrm{C}_{6}$ as shown in Fig. 3. $d_{\perp}$ and $d_{o}$ are the average lengths of $\mathrm{C}$ - $\mathrm{C}$ bonds perpendicular to the $z$ direction and of other (oblique) $\mathrm{C}$ - $\mathrm{C}$ bonds, respectively. $\alpha$ is the average angle between the oblique bonds and the $z$ axis. $R$ and $L$ are the nanotube radius and length, respectively.

\begin{tabular}{lllllll}
\hline \hline Field direction & $\mathcal{E}(Y)$ & $\mathcal{E}(Z)$ & $\mathcal{E}\left(d_{1}\right)$ & $\mathcal{E}\left(d_{2}\right)$ & $\mathcal{E}\left(d_{3}\right)$ & $\mathcal{E}(\alpha)$ \\
\hline $\mathrm{C}_{6}$ cluster & & & & & & \\
$y \leftarrow$ & -1.1 & +0.6 & +0.2 & +1.0 & -0.8 & +1.3 \\
$y \rightarrow$ & +1.2 & -0.5 & -0.1 & -0.8 & +1.1 & -1.2 \\
Field direction & $\mathcal{E}(Y)$ & $\mathcal{E}(Z)$ & $\mathcal{E}\left(d_{\perp}\right)$ & $\mathcal{E}\left(d_{o}\right)$ & & $\mathcal{E}(\alpha)$ \\
\hline
\end{tabular}

Benzene

$\begin{array}{llllll}z \uparrow & +0.0 & +0.1 & +0.1 & +0.1 & -0.1 \\ y \rightarrow & +0.1 & +0.1 & +0.1 & +0.1 & +0.1 \\ C_{14} \text { cluster } & & & & & \\ z \uparrow & & & & & \\ y \rightarrow & -0.5 & +0.7 & +0.1 & +0.1 & -0.9 \\ & +5.4 & -4.8 & +0.0 & +0.1 & +8.7\end{array}$

Phenanthrene

\begin{tabular}{llllll}
$z \uparrow$ & +0.0 & +0.2 & +0.1 & +0.1 & -0.2 \\
$y \rightarrow$ & +0.1 & +0.1 & -0.1 & +0.0 & +0.1 \\
Field direction & $\mathcal{E}(R)$ & $\mathcal{E}(L)$ & $\mathcal{E}\left(d_{\perp}\right)$ & $\mathcal{E}\left(d_{o}\right)$ & $\mathcal{E}(\alpha)$ \\
\hline
\end{tabular}

Finite $(4,4)$ CNT

$\begin{array}{llllll}z \uparrow & -0.7 & +2.9 & -0.1 & +1.4 & -3.5\end{array}$

Hydrogen terminated finite $(4,4)$ CNT

\begin{tabular}{llllll}
$z \uparrow$ & -0.5 & +2.2 & -0.1 & +1.3 & -2.5 \\
\hline \hline
\end{tabular}

thing to notice is that, although the overall deformations are in general small, as expected from the Thomas-Fermi calculation, the deformations of the pure carbon clusters are much larger than those of the corresponding hydrocarbons for both orientations ( $y$ and $z$, as given in Fig. 3) of the electric field. The $\mathrm{C}_{6}$ cluster loses its $D_{3 h}$ symmetry when the field is applied in the $y$ direction. When the field points in the positive $y$ direction, the cluster elongates $(+1.2 \%)$ along the field direction and contracts $(-0.5 \%)$ in the transverse $z$ direction. However, when the field points in the negative $y$ direction, the cluster deforms in the opposite way and its length changes by $-1.1 \%$ and $+0.6 \%$ along the $y$ and $z$ directions, respectively. The changes in the C-C bond lengths $d_{2}$ and $d_{3}$ (see Fig. 3) are substantial but have opposite signs; on the other hand, the change in $d_{1}$ is smaller. The $\mathrm{C}_{14}$ cluster elongates in the direction of the field and contracts in the trans-
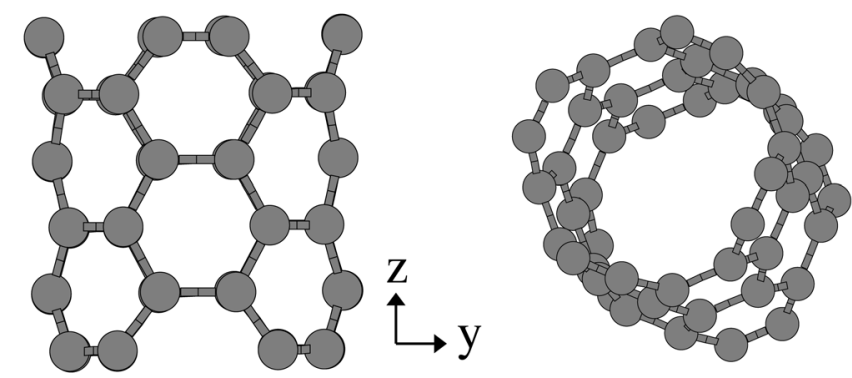

FIG. 4. Optimized geometry of the finite $(4,4)$ nanotube with 48 $\mathrm{C}$ atoms.

verse direction, for both $y$ and $z$ orientations of the field. However, the deformation is much larger when the field points in the $y$ direction. The free $\mathrm{C}_{14}$ cluster has, among others, one symmetry axis in the $y$ direction, which contains two $\mathrm{C}$ atoms, and one symmetry axis in the $z$ direction, which is perpendicular to two $\mathrm{C}-\mathrm{C}$ bonds (see Fig. 3). Therefore, the $y$ and $z$ directions are not equivalent in this cluster. An applied field in the $y(z)$ direction preserves the $y(z)$ symmetry axis of the cluster. Deformation induced by the $y$ field is easy because carbon atoms are on the two opposite ends of the elongated cluster. For the $z$ field, bonds perpendicular to the field direction appear at both ends of the deformed cluster, and the forced elongation becomes difficult.

In contrast with $\mathrm{C}_{14}$, the deformations of the benzene and phenanthrene molecules are very small. A close look into the structural modifications shows that the changes in the $\mathrm{C}-\mathrm{C}$ bond lengths are very small not only in the hydrocarbon molecules but also in the $\mathrm{C}_{14}$ cluster. This can be appreciated by comparing the changes in $d_{\perp}$ and $d_{o}$, given also in Table I. These two quantities are the average lengths of $\mathrm{C}-\mathrm{C}$ bonds perpendicular to the $z$ direction and of other (oblique) $\mathrm{C}-\mathrm{C}$ bonds, respectively. Therefore, the deformation of $\mathrm{C}_{14}$ is due mainly to the change in shape, rather than to the elongation of the bonds. This change in shape is measured in Table I by the change in the average angle $\alpha$ formed by the oblique $\mathrm{C}-\mathrm{C}$ bonds and the $z$ axis. It is interesting to notice that the deformations of the $\mathrm{C}_{6}$ and $\mathrm{C}_{14}$ clusters under an applied electric field, as calculated by DFT, agree qualitatively with the ones obtained from the simple two-dimensional ThomasFermi model.

\section{Equilibrium geometry changes in $\mathbf{C}$ nanotubes induced by an applied electric field}

We have also optimized the geometry of a finite $(4,4)$ carbon nanotube with 48 atoms using DFT in free space and with an external electric field of $0.1 \mathrm{~V} / \AA$ applied in the axial direction of the tube. The geometry in the absence of electric field is plotted in Fig. 4. The length of the nanotube is about $6 \AA$. The electrostrictive deformations undergone by the CNT upon the application of the electric field are summarized in Table I, where the relative changes in the nanotube length $(L)$ and radius $(R)$ are presented. The overall deformations arise from both changes in the $\mathrm{C}-\mathrm{C}$ bond lengths and changes in the angles between bonds. Application of this relatively weak electric field yields an axial elongation of 
$2.9 \%$ and a reduction of $0.7 \%$ of the tube radius. A similar elongation has been obtained, for a similar value of the electric field, by Guo and Guo [10] in a finite $(3,3)$ carbon nanotube with 42 atoms. The relative axial elongation of the $(4,4)$ nanotube $(+2.9 \%)$ is about two times larger than the elongation $(+1.4 \%)$ of the oblique $\mathrm{C}-\mathrm{C}$ bond lengths (which are the bonds contributing to the nanotube elongation). Therefore, the substantial elongation of the nanotube is due only partially to the elongation of the $\mathrm{C}-\mathrm{C}$ bond lengths in the axial direction of the nanotube. Half of the tube elongation is due to the deformation of the honeycomb network. This deformation can be measured by the average change $(-3.5 \%)$ of the angle $\alpha$ between the oblique C-C bonds and the $z$ axis. Our results are in contrast with Guo and Guo's [10] conclusions, which attribute the electrostrictive elongation of the tube exclusively to the change in the $\mathrm{C}-\mathrm{C}$ bond lengths. When the edge atoms of the finite $(4,4)$ nanotube are saturated with hydrogen the deformations under the applied electric field are qualitatively similar to those of the clean nanotube, but of smaller magnitude.

\section{CONCLUSIONS}

The electrostrictive response of small carbon clusters, hydrocarbon molecules, and finite carbon nanotubes to an external applied electric field has been investigated using the DFT formalism. The small hydrocarbon molecules benzene and phenanthrene are quite rigid and their deformation is negligible even for relatively intense electric fields of $1 \mathrm{~V} / \AA$. In contrast, the small $\mathrm{C}_{6}$ and $\mathrm{C}_{14}$ ring clusters experience moderate deformations under an applied field of the same intensity. The elongation of $\mathrm{C}_{14}$ in the direction of the field is due, exclusively, to its change in shape. The C-C bond lengths are not affected by the field. On the other hand, the deformation of $\mathrm{C}_{6}$ is due to both its change in shape and the change in the C-C bond lengths. Our results are in agreement with those of Tada and Watanabe [12], who found a more intense effect of the electric field in clean graphitic ribbons than on $\mathrm{H}$-terminated graphitic ribbons due to the disappearance of the dangling-bond states at the edges of the later ribbons. Moreover, we have shown that the deformations of ring carbon clusters can be explained qualitatively in terms of a simple two-dimensional Thomas-Fermi model including an external electric field. Finite carbon nanotubes experience substantial elongations induced by relatively weak $(0.1 \mathrm{~V} / \AA)$ electric fields applied in the axial direction of the nanotubes. The extent of axial elongation agrees with previous calculations [10]. However, the deformation mechanism that we have found involves the increase in the C-C bond lengths and also the change in shape of the honeycomb structure of the nanotube. This is in contrast with the mechanism proposed in a previous study [10], which only accounted for the increase of the C-C bond lengths. The fieldinduced deformations of finite nanotubes with hydrogensaturated edges are smaller than those of the finite nanotubes without hydrogen atoms: The same qualitative result that we have obtained for hydrocarbon molecules and pure carbon clusters.

Relatively intense electric fields do not deform small saturated hydrocarbon molecules whereas they induce moderate deformations in pure carbon clusters. The effect of the electric field on finite carbon nanotubes is more important, even for relatively weak fields. This indicates that the C-C bonds and the honeycomb lattice in the nanotubes are easier to deform, an effect arising from the curvature strain. Due to the absence of experimental data on the axial electrostriction of carbon nanotubes, we hope that our calculations may stimulate such experiments.

\section{ACKNOWLEDGMENTS}

This work was supported by MEC of Spain (Grant No. MAT2005-06544-C03-01), Junta de Castilla y León (Grant No. VA039A05), and the University of Valladolid. I.C. acknowledges support from MEC through the Ramón y Cajal Program. N.H.M. wishes to acknowledge that his contribution to this study was started during a visit to the University of Valladolid. He also wishes to thank Professor J. A. Alonso and Professor L. M. Nieto for generous hospitality.
[1] Y. G. Zhang, A. L. Chang, and J. Cao, Appl. Phys. Lett. 79, 3155 (2001).

[2] M. Kruger, M. R. Buitelaar, T. Nussbaumer, C. Schonenberger, and L. Forro, Appl. Phys. Lett. 78, 1291 (2001).

[3] N. S. Lee et al., Diamond Relat. Mater. 10, 265 (2001).

[4] M. Freitag, Y. Martin, J. A. Misewich, R. Martel, and P. Avouris, Nano Lett. 3, 1067 (2003).

[5] J. Cao, R. R. Lu, Y. L. Li, J. Q. Cao, D. Z. Zhu, J. L. Gong, and Z. Y. Zhu, Spectrosc. Spectral Anal. (Beijing) 23, 1079 (2003).

[6] C. W. Chen, M. H. Lee, and S. J. Clark, Nanotechnology 15, 1837 (2004).

[7] R. H. Baughman et al., Science 284, 1340 (1999).

[8] W. Lehmann, H. Skupin, C. Tolksdorf, E. Gebhard, R. Zentel, P. Kruger, M. Losche, and F. Kremer, Nature (London) 40, 447 (2001).
[9] Q. M. Zhang, V. Bharti, and X. Zhao, Science 280, 2101 (1998).

[10] W. Guo and Y. Guo, Phys. Rev. Lett. 91, 115501 (2003).

[11] Y. Guo and W. Guo, J. Phys. D 36, 805 (2003).

[12] K. Tada and K. Watanabe, Phys. Rev. Lett. 88, 127601 (2002).

[13] Y. H. Lee, S. G. Kim, and D. Tomanek, Chem. Phys. Lett. 265, 667 (1997).

[14] K. El-Hami and K. Matsushige, Ultramicroscopy 105, 143 (2005).

[15] C. Amovilli and N. H. March, Phys. Rev. A 73, 063205 (2006).

[16] T. Wakabayashi, T. Momose, and T. Shida, J. Chem. Phys. 111, 6260 (1999).

[17] D. P. Clougherty and X. Zhu, Phys. Rev. A 56, 632 (1997).

[18] F. Despa, Phys. Rev. B 57, 7335 (1998).

[19] C. Amovilli and N. H. March, Chem. Phys. Lett. 347, 459 
(2001)

[20] http://dcwww.camp.dtu.dk/campos/Dacapo

[21] B. Hammer, K. W. Jacobsen, V. Milman, and M. C. Payne, J. Phys.: Condens. Matter 4, 10453 (1992).

[22] D. Vanderbilt, Phys. Rev. B 41, 7892 (1990).

[23] J. P. Perdew, J. A. Chevary, S. H. Vosko, K. A. Jackson, M. R.
Pederson, D. J. Singh, and C. Fiolhais, Phys. Rev. B 46, 6671 (1992).

[24] K. Raghavachari and J. S. Binkley, J. Chem. Phys. 87, 2191 (1987).

[25] R. O. Jones, J. Chem. Phys. 110, 5189 (1999).

[26] R. O. Jones and G. Seifert, Phys. Rev. Lett. 79, 443 (1997). 